\title{
Neodymium: YAG Transscleral Cyclocoagulation: A Clinical Study
}

\author{
JAMES McALLISTER and COLM O'BRIEN \\ Windsor
}

\begin{abstract}
Summary
Thirty-seven eyes of 35 patients with uncontrolled glaucoma on maximum medication and after previous failed glaucoma surgery, were treated by transscleral cyclocoagulation using the free running mode of the Lasag Microrupter II Neodymium: YAG (Nd:YAG) laser. The indications, technique and results are discussed in this group of complicated glaucomatous eyes and suggests that Nd: YAG laser cyclocoagulation is a safe and effective method of lowering the intraocular pressure (IOP) and improving glaucoma control. The mean pre-treatment IOP was 41 mmHg, and the mean post-operative IOP was $22.5 \mathrm{mmHg}$. The post-operative IOP was $25 \mathrm{mmHg}$ or less in 27 of 37 eyes $(73 \%)$ after an average follow-up of eight months.
\end{abstract}

The task of controlling elevated IOP is more difficult and challenging for the secondary glaucomas than for primary open angle glaucoma. Several different techniques have been used to treat the ciliary body in an attempt to reduce aqueous humour production and thereby lower the IOP. Cyclocryotherapy, originally introduced in 1950 by Bietti, ${ }^{1}$ has become a useful method in glaucoma unresponsive to medical therapy and filtration surgery. Other methods of cyclodestruction have been described, using both the transscleral (laser and ultrasound) ${ }^{2-6}$ and transpupillary (laser) ${ }^{7}$ approach. Endophotocoagulation of the ciliary processes under direct visualisation has also been reported. ${ }^{8}$

In 1973, Beckman presented results on the pressure lowering effect of a transscleral neodymium laser in human eyes. ${ }^{3}$ Wilensky showed that a sustained lowering of IOP over a two-month period could be achieved in rabbit eyes with the Nd: YAG laser, ${ }^{9}$ and later Schwartz and others indicated that this laser could successfully lower IOP in humans. ${ }^{10-13}$ In this study we present the indications, results and complications of using transscleral $\mathrm{Nd}$ : YAG cyclocoagulation in a group of 37 eyes with uncontrolled glaucoma.

\section{Subjects and methods}

Only patients with uncontrolled secondary glaucoma were included in the study. Informed consent was obtained in each case after explaining that this form of treatment was relatively new and still under investigation. All 37 eyes (35 patients) had poorly controlled intraocular pressures despite maximum tolerated medical therapy. Twentyone eyes had previously had two or more filtration procedures, and six eyes had five or more glaucoma operations. There were 21 males and 14 females. The age range was from 14 to 78 years (mean 40 years). The mean follow-up time was eight months (range 3-30 months). The mean pre-operative IOP was 41 $\mathrm{mmHg}$ (range $25-70 \mathrm{mmHg}$ ).

From the Prince Charles Eye Unit, King Edward VII Hospital, Windsor.

Correspondence to: Mr J. A. McAllister, FRCS, FCOphth, Prince Charles Eye Unit, King Edward VII Hospital, Windsor, Berks, SL4 3DP. 
Nineteen eyes (17 patients) had glaucoma secondary to ocular inflammation, and 14 of these were associated with juvenile chronic arthritis. The remaining 18 eyes were divided into three groups:

(a) six eyes with neovascular glaucoma,

(b) six eyes had glaucoma secondary to ocular trauma,

(c) six eyes with other miscellaneous causes of secondary glaucoma.

Thirteen eyes were phakic, 22 were aphakic and two were pseudophakic. The primary indication for laser treatment was elevated IOP. Other indications included an intolerance of medication (particularly the carbonic anhydrase inhibitors) and distressing ocular pain.

Therefore, our criteria for success were

(1) A lowering of IOP to $25 \mathrm{mmHg}$ or less,

(2) A reduction in the number and frequency of medications and

(3) The elimination of ocular pain.

\section{Technique}

The patients were treated on a Lasag Microrupter II Nd: YAG laser (Thun, Switzerland). In the free running mode, the Microrupter II produces a 20 milli-second pulse of 1064 nanometers focused to a 50 micron spot size. The laser was defocused by a factor of nine which closely approximates to $3.6 \mathrm{~mm}$ behind the focus of the rotating Helium-Neon double aiming beam. Pre-operative anaesthesia was achieved with topical Proparacaine $0.5 \%$, followed by $4 \%$ Cocaine administered in cotton pledgets applied to the bulbar conjunctiva. This permits the patient to rotate the eye, and allows the operator to direct the laser beam perpendicular to the surface of the eye.

The laser applications were placed with the aiming beam focused $2-3 \mathrm{~mm}$ from the limbus (Figure). The number of laser shots varied from 12 to 36 per session, with an average of 24 . For the majority of eyes, the laser shots were placed between two to 10 o'clock positions, avoiding the superior quadrant, and in general they were concentrated in the lower $180^{\circ}$. Eight eyes had laser shots placed around the $360^{\circ}$. No contact lens was used.

Twelve eyes were treated with low energy levels (approximately $2-2.5 \mathrm{~J}$ ). All other treatments were with high energy levels of between $4-5 \mathrm{~J}$.
The laser applications were normally visible by a blanching of the conjunctiva or transient closure of the superficial conjunctival or episcleral vessels. During treatment, patients were aware of the laser pulses but in general did not sustain more than a mild preoperative discomfort.

After the procedure, analgesia was provided as necessary and paracetamol tablets were sufficient to control the pain. Post-operative medications included Dexamethasone $0.1 \%$, two hourly for 48 hours, followed by four times a day for one week. All glaucoma medications were maintained for two months and then the decision to discontinue was made on the basis of glaucoma control. Initially, all patients were admitted to the ward for careful monitoring of the IOP over a two-day period, and later reviewed at one week, two-three weeks and subsequently one-two monthly thereafter.

As the study progressed, we noted that none of the eyes had a pressure spike in the immediate 48 hours after treatment and later in the study, patients were allowed home on the day of the procedure. Patients were retreated for two reasons; firstly, if they showed little or no response to the initial treatment; secondly, if the IOP began to rise to an unacceptably high level after an initially good response to the treatment. As a result the timing of the retreatments varied from one to 20 months. Both the energy level and the number of applications used in the retreatments were titrated against the level of the IOP of the type of response to the initial treatment regime.

\section{Results}

The mean pre-treatment IOP was $41 \mathrm{mmHg}$. The mean IOP at the last clinic visit (average follow up 8 months) was $22.5 \mathrm{mmHg}$. At this time, 27 eyes $(73 \%)$ had an IOP of $25 \mathrm{mmHg}$ or less. At 2-3 months post-treatment, the success rate was slightly higher with 31 eyes $(84 \%)$ having ocular pressures at $25 \mathrm{mmHg}$ or less. Thirty of the 37 eyes had pre-treatment pressures greater than $30 \mathrm{mmHg}$, and at the end of the study period 30 of 37 eyes had pressures less than $30 \mathrm{mmHg}$. Overall 34 of the 37 eyes showed some lowering in the IOP level.

Twenty-three patients were able to discon- 


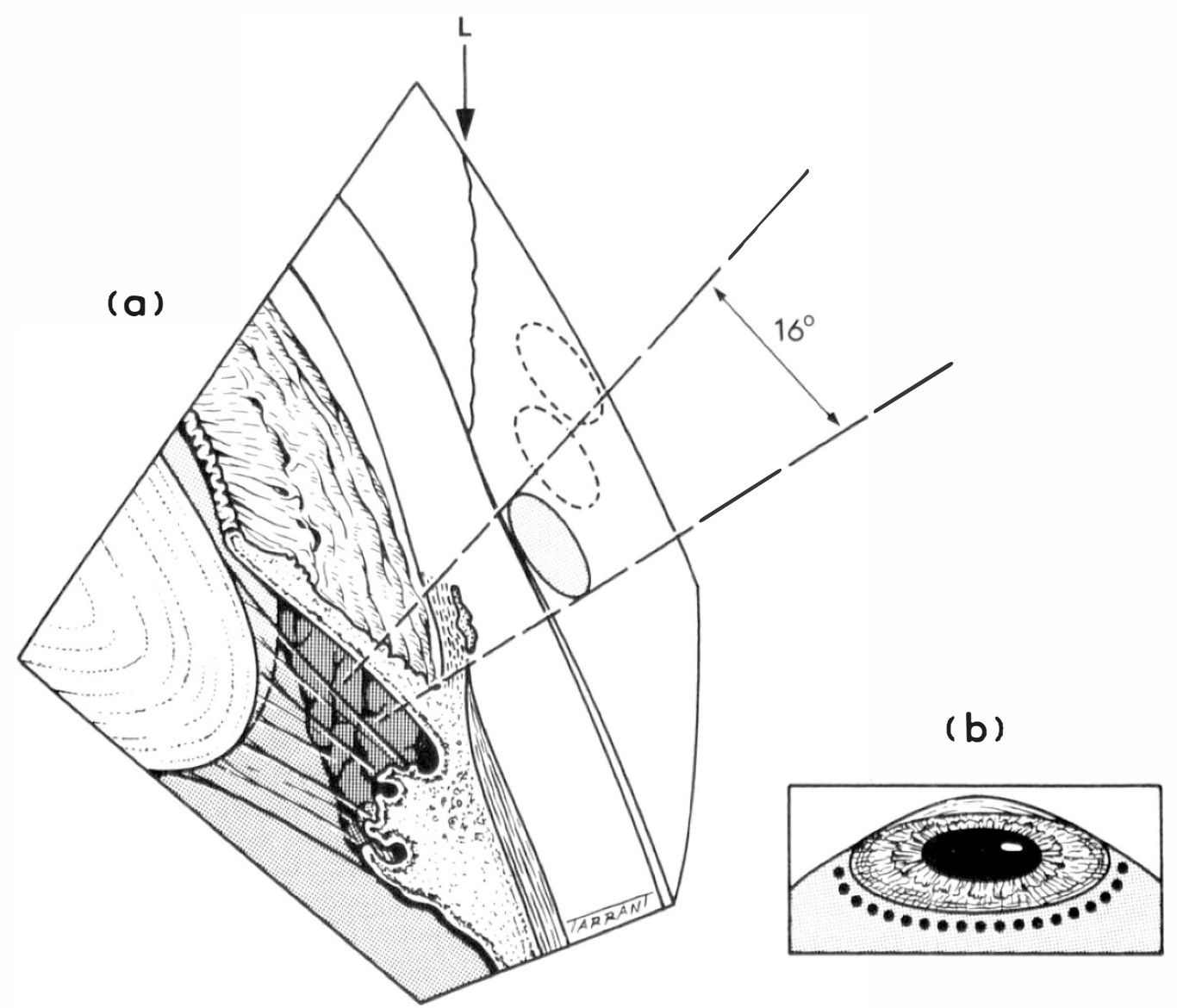

Figure

tinue or reduce their dosage or carbonic anhydrase inhibitors. Seven of the eight patients with pre-operative pain had complete resolution of the pain while the eighth patient continued to experience pain despite controlled IOP. The cause of pain in this case was related to corneal ulceration, which was a complication of radiation therapy for an olfactory neuroblastoma and not related to the laser treatment. This eye was later enucleated because of corneal perforation and persistent pain.

The six eyes with neovascular glaucoma had successful lowering of the IOP below 25 $\mathrm{mmHg}$. Four of the six eyes with trauma related glaucoma had successful pressure reduction, and one had no response. The last eye of this group showed a pressure reduction from $70 \mathrm{mmHg}$ to $40 \mathrm{mmHg}$ and had relief of pain in a blind eye, and could therefore be regarded as a qualified success. Six of the 12 eyes treated with low energy levels (2-2.5J) were successful. Four of the six failures were successfully retreated with higher energy levels (4-5J), but two eyes failed to respond to the higher energy treatment. One of the eyes progressed to a further drainage procedure, the other was an NPL eye and had no further treatment.

\section{Complications}

Conjunctival injection and oedema were seen adjacent to the site of the laser lesions. The injection and oedema resolved within fourfive days of the treatment. Anterior uveitis was observed in all cases and this responded well to topical corticosteroids in all but two eyes. Two eyes with neovascular glaucoma had a significant anterior chamber reaction with the development of small (1-2 mm) 
hypopyons. These eyes required 2-3 weeks intensive topical corticosteroid treatment to control the inflammation. We have not seen any evidence of cataract formation in eyes with clear lenses prior to treatment. Two eyes with pre-existing lenticular opacities showed progression of cataract formation. To date we have not encountered scleral thinning, persistent hypotony or phthisis bulbi. We did not observe an early pressure spike develop in any of the patients admitted to the ward for observation after treatment. One diabetic patient developed macular oedema nine months following the laser procedure and it is difficult to know whether this occurred as a very late complication of treatment or whether it was related to the diabetic retinopathy which the patient had.

Two eyes were enucleated during the follow-up period despite having well controlled ocular pressures. One eye had corneal ulceration leading to perforation following radiotherapy for an olfactory neuroblastoma. The other eye developed glaucoma secondary to a penetrating ocular injury. Laser treatment successfully reduced the IOP in this blind eye, but the patient, a young female, elected to have an enucleation for cosmetic reasons.

Two of the eyes showed deterioration of visual acuity during the study. One was a diabetic that lost vision from $6 / 9$ to counting fingers nine months after successful IOP lowering from macular oedema. The other eye deteriorated from hand movements to NPL and this eye was the eye discussed above that perforated from corneal ulceration.

\section{Discussion}

In this study of transscleral Nd: YAG cyclocoagulation, 27 of 37 eyes $(73 \%)$ had an IOP of $25 \mathrm{mmHg}$ or less at the final follow-up clinic visit. The pre-operative mean IOP of 41 $\mathrm{mmHg}$ was reduced to a mean of $22.5 \mathrm{mmHg}$ at an average follow-up of eight months. When viewed in the context of uncontrolled secondary glaucoma, the pressure lowering effect of this technique is seen to be very effective, and our results compare favourably with other reports of this procedure. ${ }^{10-13}$ It is important to note that no pressure spike was observed in the 48 hours after treatment. Furthermore, many of the patients were able to reduce or discontinue using carbonic anhydrase inhibitors, and seven of the eight eyes with ocular pain had complete resolution of the pain.

All six eyes with neovascular glaucoma and four of the six eyes with traama related glaucoma had successful lowering of IOP to 25 $\mathrm{mmHg}$ or less. Although less effective in the uveitic group, the majority of these eyes had some reduction in IOP. The technique may be considered useful in this setting, particularly for the younger cases of juvenile chronic arthritis, when repeated general or retrobulbar anaesthesia for filtration surgery and cyclocryotherapy is often necessary.

Six of the 12 eyes in the low energy group required retreatment. Four of the six eyes retreated with higher energy levels were successful in terms of lowering the IOP. This suggests that for these eyes, initial treatment with higher energy levels may well have been successful. However, these eyes might also have responded satisfactorily to a second treatment of lower energy levels. Investigators will have to address the issue of how much energy is required to achieve the desired clinical effect. Histological evidence using post-mortem eyes suggest that energy levels as high as $7-8 \mathrm{~J}$ are necessary. ${ }^{14,15}$

One of the advantages of this technique is the relative ease of use especially as it is performed as an outpatient procedure. We have used topical anaesthesia alone since we started to treat patients with uncontrolled secondary glaucoma with this technique. Almost all patients were aware of each laser application but very few of them found it too painful or distressing to warrant stopping the treatment session. The other reports of Nd:YAG cyclocoagulation have used retrobulbar anaesthesia in their methodology, which in itself is not without potentially serious side effects.

Comparing the success of different cyclodestructive procedures is complicated by varying definitions. One group may accept a final IOP of $21 \mathrm{mmHg}$ (or $25 \mathrm{mmHg}$ ) without any additional medications, while others may include a sub-group of qualified success where medication is still required. The most important gauge of success is that of halting the progression of disease itself and therefore further 
visual field loss. This is the only true measure of success but is the most difficult to measure. Cyclocryotherapy has been performed for many years to treat various types of uncontrolled glaucoma and the use of the standardised technique has been encouraged. ${ }^{16}$ It has been used successfully in advanced primary open angle glaucoma, aphakic open angle glaucoma and to a lesser extent in neovascular glaucoma (NVG). ${ }^{16,17}$ More recently, high intensity focused ultrasound has been used to treat refractory glaucoma, and Burgess has reported that $65 \%$ of patients maintained an IOP of $25 \mathrm{mmHg}$ or less after one year. ${ }^{18}$

When judging the success of a procedure, the frequency and severity of the complications that may occur must also be considered. With this difficult group of uncontrolled glaucoma, where no intervention is almost certain to result in blindness, a higher complication rate may be more acceptable than in most forms of ocular surgery. Serious complications seen with cyclocryotherapy include hypotony, macular oedema, choroidal haemorrhage, or retinal detachment but the most devastating is phthisis bulbi. Phthisis is more likely to occur in NVG and in those treated with more than $300^{\circ}$ freezing of the ciliary body, and in those with retreatment performed within one month of the initial procedure. Also, up to $50 \%$ of patients with NVG show progression to no light perception vision following cyclotherapy. For this reason, it has been recommended that cyclotherapy not be employed in NVG with useful vision. The major complications seen with ultrasound treatment include hypotony, phthisis bulbi, corneal problems and macular phthisis oedema. ${ }^{18}$ Scleral thinning has been described and occurs more commonly in children than in adults. This may lead to staphyloma formation. ${ }^{19.20}$ In the other detailed studies of Nd:YAG transscleral cyclocoagulation reported to date, the only serious complication was the occurrence of vitreous haemorrhage. This may well have been related to the underlying pathology in these eyes (NVG), and not a direct side effect of the treatment. In the early reports of this technique, no patient progressed to no light perception or phthisis bulbi. However, Trope has recently reported that three of 29 eyes treated with Nd:YAG cyclocoagulation became phthisical. ${ }^{21}$ The background and treatment details of these eyes will need to be reviewed carefully for possible reasons which may have contributed to the development of phthisis. None of our patients developed phthisis and all but two of the patients retained their pretreatment visual acuity.

In summary, Nd:YAG transscleral cyclocoagulation appears to be a useful alternative form of therapy in the management of complicated glaucomas with elevated IOP.

\section{References}

${ }^{1}$ Beitti G: Surgical intervention of the ciliary body; new tends for the relief of glaucoma. J Am Med Assn 1950, 142: 889-97.

${ }^{2}$ Weekers R, Lavergne G, Watillon M, Gilson M, Legros AM: Effects of photocoagulation of ciliary body upon ocular tension. Am J Ophthalmol 1961, 52: 156-63.

${ }^{3}$ Beckman $\mathrm{H}$ and Sugar S: Neodymium laser cyclocoagulation. Arch Opthalmol 1973, 90: 27-8.

${ }^{4}$ Peyman GA, Conway MD, Raichland M, Lin J: Histopathologic studies of transscleral argon-krypton photocoagulation with an exolaser probe. Ophthalmic Surg 1984, 15: 496-501.

${ }^{5}$ Beckman $\mathrm{H}$ and Waeltermann $\mathrm{J}$ : Transscleral ruby laser cyclocoagulation. Am J Ophthalmol 1984, 98: 788-95.

${ }^{6}$ Coleman DJ, Lizzi FL, Driller J et al: Therapeutic ultrasound in the treatment of glaucoma II. Clinical applications. Ophthalmology 1985, 92: 347-53.

${ }^{7}$ Lee PF: Argon laser photocoagulation of the ciliary processes in cases of aphakic glaucoma. Arch Ophthalmol 1979, 97: 2135-8.

${ }^{8}$ Patel A, Thompson JT, Michels RM, Quigley HA: Endolaser treatment of the ciliary body for uncontrolled glaucoma. Ophthalmology 1986, 93: 825-30.

${ }^{9}$ Wilensky JT, Welch D, Mirolovich M: Transscleral cyclocoagulation using a neodymium: YAG laser. Ophthalmic Surg 1985; 16: 95-8.

${ }^{10}$ Schwartz LW and Moster MR: Neodymium: YAG laser transscleral cyclodiathermy. Opthalmic Laser Ther 1986, 1: 135-41.

${ }^{11}$ Crylin MN, Beckman H, Czedik C: Neodymium: YAG transscleral cyclocoagulation for severe glaucoma. (ARVO Abstracts) Invest Ophthalmol Vis Sci 1986, 26: (Suppl): 157.

${ }^{12}$ Devenyi RG, Trope GE, Hunter WH, Badeeb O: Neodymium: YAG transscleral cyclocoagulation in human eyes. Ophthalmology 1987, 94: 1519-22.

${ }^{13}$ Klapper RM, Wandel T, Donnerfeld E, Perry HD: Transscleral neodymium: YAG thermal cyclophotocoagulation in refractory glaucoma. A preliminary report. Ophthalmology 1988, 95: 719-22. 
${ }^{14}$ Fankhauser F, van der Zypen E, Kwasniewska S, Rol P, England C: Transscleral cyclophotocoagulation using a neodymium: YAG laser. Ophthalmic Surg 1986, 17: 94-100.

${ }^{15}$ Hampton C and Shields MB: Transscleral neodymium: YAG cyclophotocoagulation. A histological study of human autopsy eyes. Arch Ophthalmol 1988, 106: 1121-3.

${ }^{16}$ Bellows AR: Cyclocryotherapy for glaucoma. Int Ophthalmol clin 1981, 21(1): 99-111.

${ }^{17}$ Caprioli J, Strang SL, Spaeth GL, Poryzees EH: Cyclocryotherapy in the treatment of advanced glaucoma. Ophthalmology 1985, 92: 947-53.
${ }^{18}$ Burgess SEP, Silverman RH, Coleman DJ, et al: Treatment of glaucoma with high-intensity focused ultrasound. Ophthalmology 1986, 93: 831-38.

${ }^{19}$ Wilensky JT: Staphyloma formation as a complication of ultrasound treatment in glaucoma. Arch Ophthalmol 1985; 103: 113.

${ }^{20}$ Coleman JD: in reply. Arch Ophthalmol 1985, 103: 113.

${ }^{21}$ Trope GE and Ma S: Mid-term effects of Nd:YAG transscleral cyclocoagulation ARVO Abstracts. Invest Ophthalmol Vis Sci 1989, 30 (Suppl): 281. 